\title{
Obstructive Sleep Apnea Syndrome does not increase the cardiovascular risk in a naïve population of southern Italy
}

\author{
Pierluigi Carratù ${ }^{1}$, Agostino di Ciaula ${ }^{2}$, Silvano Dragonieri ${ }^{3}$, Teresa Ranieri $^{3}$, Marco \\ Ciccone $^{4}$, Piero Portincasa ${ }^{2}$, and Onofrio Resta ${ }^{3}$ \\ ${ }^{1}$ Division of Internal Medicine "A. Murri" \\ ${ }^{2}$ Division of Internal Medicine "A. Murri" , Department of Biomedical Sciences and \\ Human Oncology, University of Bari " Aldo Moro", Bari, Italy Piazza G. Cesare 12, Bari, \\ Italy Bari, IT 70124 \\ ${ }^{3}$ Institute of Respiratory Diseases, University of Medicine, Bari, Italy \\ ${ }^{4}$ Institute of Cardiovascular Diseases, University of Medicine, Bari, Italy
}

March 16, 2021

\begin{abstract}
Background Obstructive Sleep Apnea (OSA) is a worldwide increasing syndrome, which, by promoting endothelial dysfunction, contributes to extend the cardiovascular risk. We evaluated the cardiovascular risk in a group of OSA patients. Methods A total of 185 OSA subjects (19 normal weight, 57 overweight, 109 obeses), seen at the Ambulatory of Sleep Disorders, during one year, entered the study. We assessed anthropometric features, polysomnographic findings, cardiovascular risk factors, smoking habit, Pulmonary Function Test, Arterial Blood Gas Analysis, Epworth Questionnaire, and Charlson Co-morbidities Index (CCI). Subjects were divided into three groups, according to their BMI: individuals with BMI [?]30 Kg/m2 (Group $1 \mathrm{n}=109$, mean age $61 \pm 1 ; 74.3 \%$ males), individuals with BMI ranging from 25.0 to $29.9 \mathrm{Kg} / \mathrm{m} 2$ defined as overweight subjects (Group $2 \mathrm{n}=57$, mean age $58.8 \pm 1.4 ; 77 \%$ males) and subjects with a BMI ranging from 18.5 to $24.9 \mathrm{Kg} / \mathrm{m} 2$ defined normal weight subjects (Group $3 \mathrm{n}=19$, mean age $54.2 \pm 2.3 ; 64,2 \%$ males). Results In the whole population, the percentage cardiovascular risk was weakly related with BMI $(\mathrm{r}=0.33 ; \mathrm{P}<0.001)$, but not with AHI. The cardiovascular risk was strictly related to the obesity $(\mathrm{p}<0.00002)$, while the Epworth Questionnaire score and the Charlson Co-morbidity Index were respectively statistically higher in the group of obese individuals $(\mathrm{p}=0.006, \mathrm{p}=0.00002)$ than in the other 2 Groups. When AHI values were stratified in tertiles, the percentage cardiovascular risk did not vary with increasing AHI values (Figure 2). Conclusions Further studies are required to investigate the pivotal role of inflammation due to obesity, and underlying increased cardiovascular risk in OSA patients.
\end{abstract}

Obstructive Sleep Apnea Syndrome does not increase the cardiovascular risk in a naïve population of southern Italy

Pierluigi Carratù, Agostino Di Ciaula, Silvano Dragonieri*, Teresa Ranieri*, Marco Matteo Ciccone $^{\circ}$, Piero Portincasa, Onofrio Resta*.

Division of Internal Medicine, Clinica Medica "A. Murri", Department of Biomedical Sciences and Human Oncology, University of Bari ""Aldo Moro", Bari, Italy

*Institute of Respiratory Disease, University of Medicine "Aldo Moro", Bari, Italy

${ }^{\circ}$ Institute of Cardiovascular Disease, University of Medicine, "Aldo Moro", Bari, Italy

Corresponding Author: Pierluigi Carratù, Division of Internal Medicine, Clinica Medica "A. Murri", Department of Biomedical Sciences and Human Oncology, University of Bari "'Aldo Moro", Bari, Italy. Piazza G. 
Cesare 12, 70122, Bari, Italy.

e-mail:pierluigi.carratu@uniba.it/ Fax number 0805592907.

Abstract

Background Obstructive Sleep Apnea (OSA) is a worldwide increasing syndrome, which, by promoting endothelial dysfunction, contributes to extend the cardiovascular risk. We evaluated the cardiovascular risk in a group of OSA patients.

Methods A total of 185 OSA subjects (19 normal weight, 57 overweight, 109 obeses), seen at the Ambulatory of Sleep Disorders, during one year, entered the study. We assessed anthropometric features, polysomnographic findings, cardiovascular risk factors, smoking habit, Pulmonary Function Test, Arterial Blood Gas Analysis, Epworth Questionnaire, and Charlson Co-morbidities Index (CCI). Subjects were divided into three groups, according to their BMI: individuals with BMI [?] $30 \mathrm{Kg} / \mathrm{m}^{2}$ (Group $1 \mathrm{n}=109$, mean age 61 +- 1; $74.3 \%$ males), individuals with BMI ranging from 25.0 to $29.9 \mathrm{Kg} / \mathrm{m}^{2}$ defined as overweight subjects (Group $2 \mathrm{n}=57$, mean age $58.8+-1.4 ; 77 \%$ males) and subjects with a BMI ranging from 18.5 to $24.9 \mathrm{Kg} / \mathrm{m}^{2}$ defined normal weight subjects (Group $3 \mathrm{n}=19$, mean age $54.2+-2.3 ; 64,2 \%$ males).

Results In the whole population, the percentage cardiovascular risk was weakly related with BMI ( $\mathrm{r}=0.33$; $\mathrm{P}<0.001)$, but not with AHI. The cardiovascular risk was strictly related to the obesity $(\mathrm{p}<0.00002)$, while the Epworth Questionnaire score and the Charlson Co-morbidity Index were respectively statistically higher in the group of obese individuals $(\mathrm{p}=0.006, \mathrm{p}=0.00002)$ than in the other 2 Groups. When AHI values were stratified in tertiles, the percentage cardiovascular risk did not vary with increasing AHI values (Figure 2).

Conclusions Further studies are required to investigate the pivotal role of inflammation due to obesity, and underlying increased cardiovascular risk in OSA patients.

Keywords: Obstructive Sleep Apnea, Obesity, BMI, Cardiovascular Risk, Polysomnography, Continuous Positive Airway Pressure.

Introduction

The cardiovascular risk is defined by WHO/ISH by risk prediction charts, which indicate 10-year risk of a fatal or nonfatal major cardiovascular event (myocardial infarction or stroke), according to age, sex, blood pressure, smoking status, total and HDL blood cholesterol, presence or absence of diabetes mellitus, and anti-hypertensive treatment (1)

Obstructive Sleep Apnea (OSA) is a worldwide increasing syndrome and is a persistent long term risk factor for several cardio-pulmonary diseases (2). In particular, in patients with OSA, chronic upper airways inflammation constantly promotes a systemic endothelial dysfunction $(3,4)$ worsens the cardio-pulmonary activity, thus, affecting the left and the right heart performance (5) increases risk of cardiovascular events, over time (6).

Obesity plays a well established pivotal role in the evolution of the cardiovascular risk in patients with OSA (7). Indeed, in obese individuals, the eccentric ventricular hypertrophy and the diastolic heart failure are responsible for developing cardio-myopathy, a well-recognized complication in severe obese patients (8). The augmented left ventricular filling pressure, transmitted into the pulmonary venous system, improves pulmonary venous pressures leading to increased pulmonary vascular resistance (9). Obesity is also linked with elevated serum hs-CRP levels in patients with sleep-disordered breathing (10), further contributing to chronic systemic inflammation. Thus, the complex interplays among OSA, insulin resistance, hypercholesterolemia, obesity, chronic systemic inflammation and elevated left ventricular filling is responsible for endothelial dysfunction, with Pulmonary Hypertension $(\mathrm{PH})$ and finally increasing cardiovascular risk in affected subjects $(11,12,13)$. However, besides the combined role of these factors, it is still under debate if the severity of OSA can act per se, as an independent factor in determining the extent of the cardiovascular risk in obese patients (14). 
In the present study, we depicted the cardiovascular risk of a consecutive population of OSA patients enrolled at the Sleep Ambulatory, and studied for anthropometric features, cardiovascular risk, and Polysomnographic findings.

Subjects and Methods

Patients

OSA patients entering, during the year 2016, the Ambulatory of Sleep Disorders of the Institute of Respiratory Diseases of the University of Bari were retrospectively evaluated. In enrolled subjects, we recorded anthropometric features, smoking habits, Pulmonary Function Test, polysomnographic findings, data from Arterial Blood Gas Analysis.

Daytime sleepiness was measured by the Epworth Questionnaire, and the death risk linked with comorbidities was assessed by the Charlson Co-morbidities Index (CCI). The 10-year risk of heart disease or stroke was calculated using the ASCVD algorithm (15).

We measured body weight $(\mathrm{kg})$, height $(\mathrm{m})$ and calculated BMI, i.e., Quetelet's index as kilograms divided by meter squared $(\mathrm{kg} / \mathrm{m} 2)$. BMI ranging from 18.5 to $24.9 \mathrm{~kg} / \mathrm{m} 2$ defined normal weight subjects. A BMI ranging from 25.0 to $29.9 \mathrm{~kg} / \mathrm{m} 2$ defined overweight subjects, while a BMI [?] $30 \mathrm{~kg} / \mathrm{m} 2$ defined patients with obesity.

\section{Pulmonary Function Test}

Pulmonary function tests were performed by spirometry (PK Morgan Ltd; Gillingham, UK). The equipment was calibrated daily using a 3-l syringe, and the analysis was performed in accordance to the guidelines of the American Thoracic Society (16). The best of three reproducible values was expressed as a percentage of the predicted normal value.

\section{Sleep Study}

In the sleep laboratory of the Institutes of Respiratory Diseases of the University of Bari, all patients were evaluated for sleep disordered breathing during one night. They were monitored continuously for about $8 \mathrm{~h}$ using a portable cardiorespiratory monitoring (POLYMESAM(r), MAP, Martinsried, Germany). The POLYMESAM(r) (PM) device consists of a recorder, to which multiple sensors are linked for the detection of the following signals: oxy-hemoglobin saturation (by a finger sensor), heart rate (derived from three electrocardiogram (ECG) electrodes placed on the chest), snoring sound (by a microphone placed on the thyroid cartilage), body posture oro-nasal airflow (by a threefold thermocouple sensor for both nostrils and mouth), and thoracic and abdominal movements (by stretch belts). Apnea was identified if the airflow was absent or nearly absent for at least $10 \mathrm{~s}$. Hypopnea, was considered when there was a decrease of airflow for at least 10 seconds in respirations, a 30-percent reduction in ventilation, and a decrease in oxygen saturation.

\section{Statistics}

Data are presented as mean +- standard error (SEM) or as percentages. One-way analysis of variance (ANOVA) assessed inter-group differences. Differences between two groups were tested by Student's t-test for unpaired data. The Chi-square test was used to compare proportions. The Pearson correlation coefficient was used for correlations. Results were considered significant at the $5 \%$ critical level $(\mathrm{P}<0.05)$. Statistical analyses were performed with NCSS10 Statistical Software (NCSS, LLC. Kaysville, Utah, USA).

\section{Study Approval}

Since the study was retrospective, the patients did not sign any informed consent, while the Local Ethic Committee approved the study number 1089, July the31 ${ }^{\text {st }} 2009$, with note prot n. 24961/DS 03/26/2009, and approved the study number prot. N. 44696/DS 06-03-2009 n.527/DG/2004modified by the protocol n. $828 / \mathrm{DG} / 2007$.

Table 1 


\begin{tabular}{lllll}
\hline & Normal weight & Overweight & Obese & $\mathrm{P}$ \\
\hline $\mathrm{n}$. & 19 & 57 & 109 & \\
Males & 12 & 44 & 81 & \\
Age (year) & $54.2 \pm 2.3$ & $58.8 \pm 1.4$ & $61 \pm 1.0^{*}$ & 0.02 \\
BMI (Kg/m2) & $23.1 \pm 1.0$ & $27.6 \pm 0.6^{*}$ & $35.9 \pm 0.4^{* \circ}$ & 0.0000 \\
Smokers (\%) & $15.8 \%$ & $15.8 \%$ & $74.3 \%$ & $<0.05$ \\
Systemic Hypertension (\%) & $31.6 \%$ & $35 \%$ & $59.6 \%$ & $<0.05$ \\
Type 2 Diabetes (\%) & $5.3 \%$ & $3.5 \%$ & $27.5 \%$ & $<0.05$ \\
Total cholesterol (mg/dL) & $200.6 \pm 3.0$ & $202.4 \pm 1.7$ & $218.1 \pm 1.2^{* \circ}$ & 0.00001 \\
HDL cholesterol (mg/dL) & $61.3 \pm 1.8$ & $60.0 \pm 1.0$ & $53.9 \pm 0.8^{* \circ}$ & 0.0001 \\
AHI & $34.8 \pm 3.1$ & $35.5 \pm 1.8$ & $43.3 \pm 1.3^{* \circ}$ & 0.0006 \\
Cardiovascular risk (\%) & $10.2 \pm 3.1$ & $13.7 \pm 1.8$ & $22.3 \pm 1.3^{* \circ}$ & 0.00002 \\
Epworth score & $8.3 \pm 3.7$ & $14,1 \pm 5.6$ & $18.6 \pm 5.2$ & 0.006 \\
Charlson Co-morbidities Index & $2.4 \pm 1.2$ & $3.5 \pm 1.7$ & $4.8 \pm 2.0$ & 0.0002 \\
\hline
\end{tabular}

${ }^{*} \mathrm{P}<0.05$ vs normal weight; ${ }^{\circ} \mathrm{P}<0.05$ vs overweight

\section{RESULTS}

A total of 185 OSA subjects (19 normal weight, 57 overweight, 109, obese) entered the study. Anthropometric data and sleep study from normal weight, overweight and obese subjects are depicted in Table1.

AHI values were higher in obese $(43.3 \pm 1.3)$ than in lean $(34.8 \pm 3.1)$ and overweight subjects $(35.5 \pm 1.8$, $\mathrm{P}<0.01$ ANOVA). Overall, a direct relationship was evident between AHI and BMI (Figure1). Smokers were higher in obese group $(\mathrm{p}<0.05)$; Systemic Hypertension was statistically more significant among obese than in leans and overweight patients $(\mathrm{p}<0.005)$. Type 2 diabetes was more represented among obese patients $(\mathrm{p}<0.05)$. Blood total and HDL cholesterol were, respectively, statistically significant higher and lower in obese patients $(\mathrm{p}<0.00001 ; \mathrm{p}<0.0001)$.

The cardiovascular risk was strictly related to the obesity $(\mathrm{p}<0.00002)$, while the Epworth Questionnaire score and the Charlson Co-morbidity Index were respectively statistically higher in the group of obese individuals $(\mathrm{p}=0.006, \mathrm{p}=0.00002)$ than in the other 2 Groups.

In the whole population, the percentage cardiovascular risk was weakly related with $\mathrm{BMI}(\mathrm{r}=0.33 ; \mathrm{P}<0.001)$, but not with AHI.

Figure 1 shows the percentage of cardiovascular risk in the whole population which was weakly related with BMI $(\mathrm{r}=0.33 ; \mathrm{P}<0.001)$, but not with AHI. 


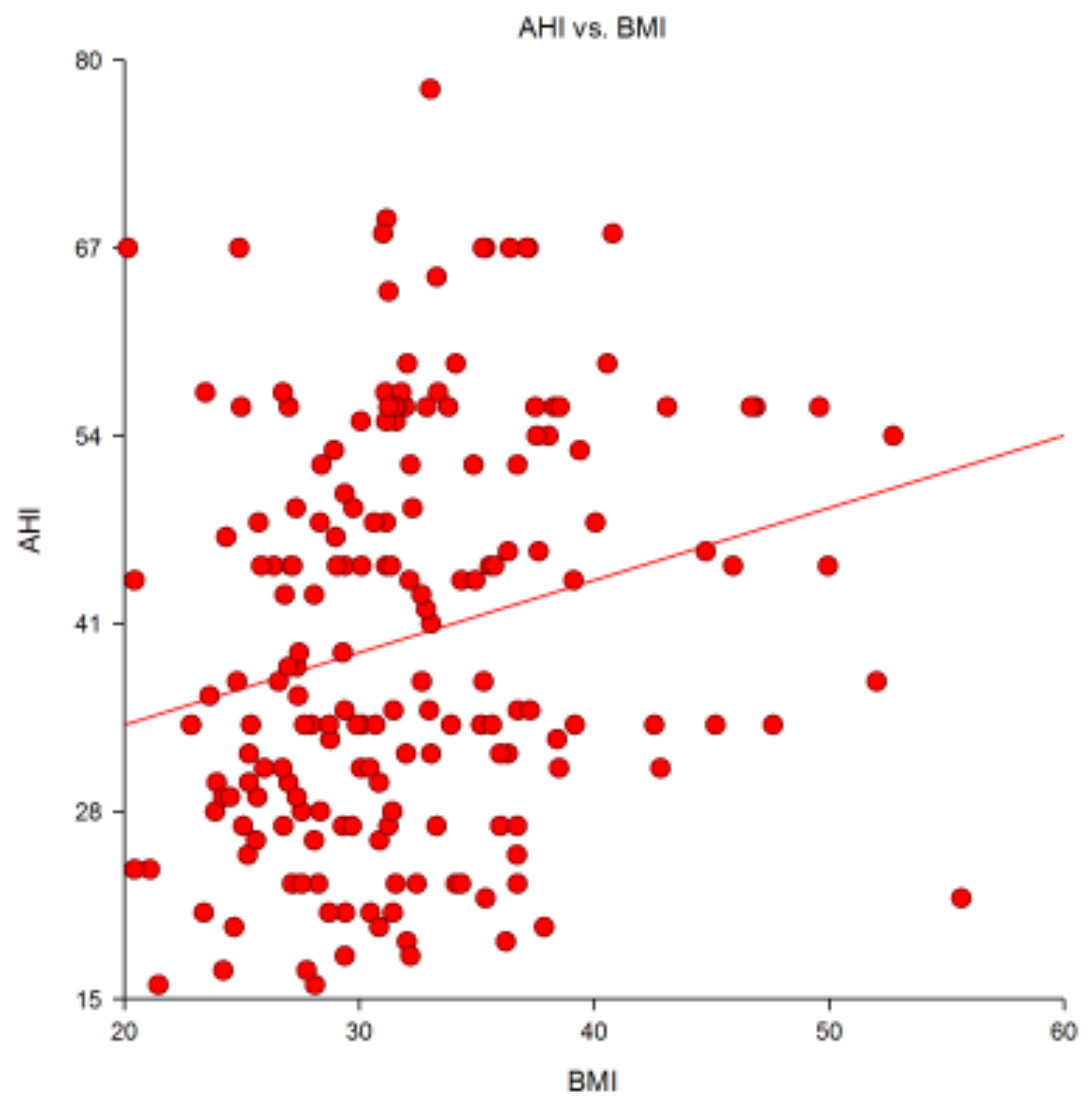

When AHI values were stratified in tertiles, the percentage cardiovascular risk did not vary with increasing AHI values (Figure2 ). 


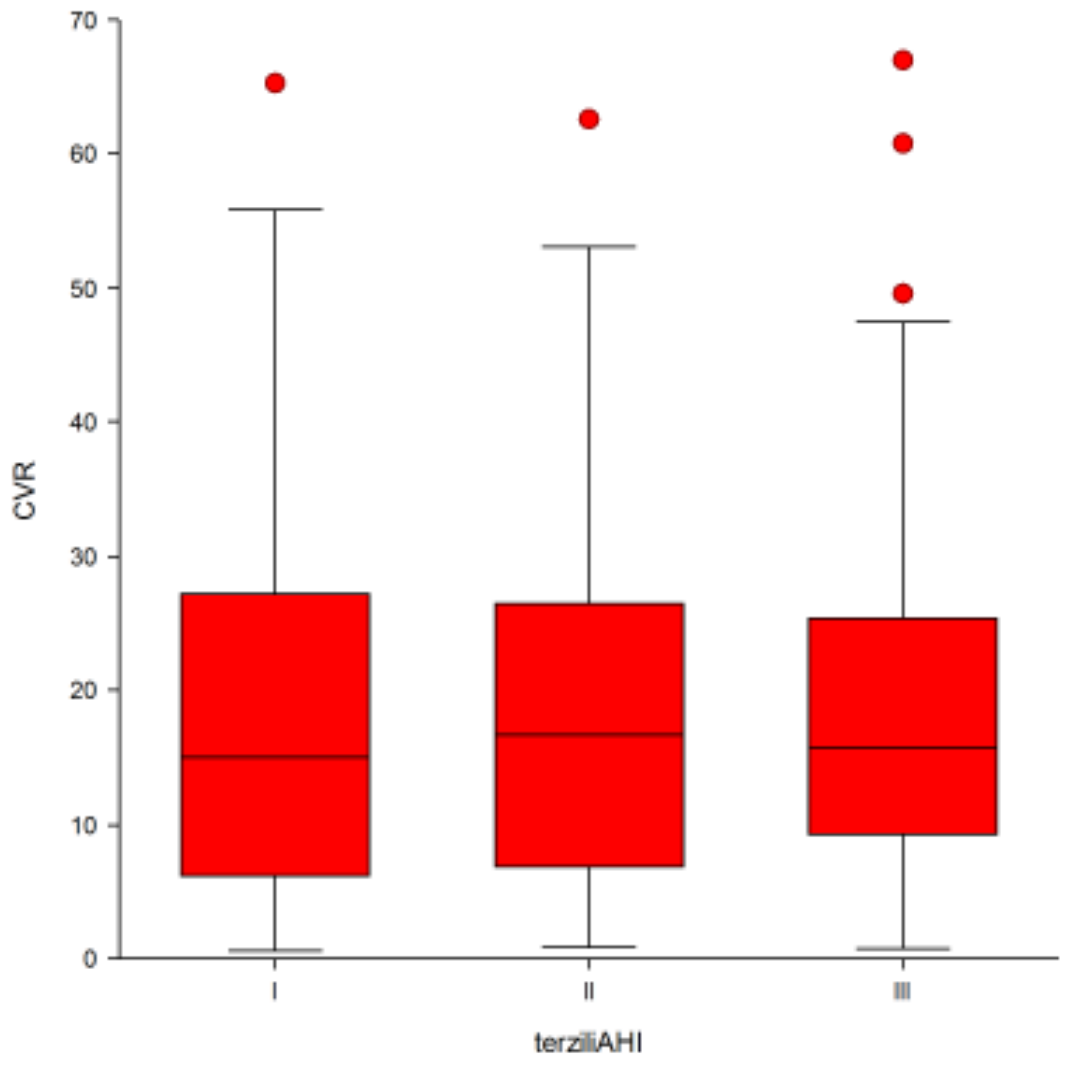

\section{Discussion}

Obstructive sleep apnoea (OSA) consists of repeated episodes of extrathoracic upper airway obstruction associated with intra-arterial hypoxaemia and hypercapnia (17). OSA, as a chronic condition is also responsible for endothelial dysfunction, with early atherosclerosis plaque evidence, and rapid increase of cardiovascular risk (6). Since, even very recently, several studies have pointed out the role of the degree of OSA, as factor likely responsible for increasing cardiovascular risk (18), on the other hand, by the contrast, recent evidences, derived by the results of an observational 1 year study, did not establish any correlation between severe obstructive sleep apnea syndrome and increased cardiovascular risk, as well as augmented cardiovascular co-morbidities (19). This debate has involved, over time, several investigators, who hypothesized both possible scenarios. Thus, it remains under discussion the primary role of OSA for developing increased cardiovascular risk in these subjects (20).

A validate assessment established that the metabolic syndrome commonly coexist with OSA, and obesity is perhaps the strongest predictor of OSA with $40-60 \%$ of obese subjects suffering from sleep disorders breathing (21). The burden of obesity is worrisome; the obesity prevalence is globally increasing, affecting 10 to $25 \%$ of the world population and is associated with a wide range of abnormalities, including elevated systemic as well as elevated pulmonary artery pressures (22), both sustained by systemic inflammation $(7,10)$. Furthermore, obesity is characterized by increased production and secretion of a wide range of inflammatory molecules, including TNF-alpha and interleukin-6, which have both, local effects on adipose tissue physiology, and systemic effects on other organs, such as the cardiovascular system (12). Thus, contributing to activate various inflammatory cells, such as lymphocytes and monocytes, leading to over- 
expression of pro-inflammatory mediators, that may establish irreversible endothelial dysfunction. The latter observation has been recently demonstrated in a rabbit model (23).

In the present study, we found In the whole OSA population, the percentage of cardiovascular risk was weakly related with BMI $(\mathrm{r}=0.33 ; \mathrm{P}<0.001)$ but not with AHI.

AHI values were higher in obese $(43.3 \pm 1.3)$ than in lean $(34.8 \pm 3.1)$ and overweight subjects $(35.5 \pm 1.8$, $\mathrm{P}<0.01$ ANOVA). Overall, a direct relationship was evident between AHI and BMI. When AHI values were stratified in tertiles, the percentage cardiovascular risk did not vary with increasing AHI values.

Then, we assumed that the degree of OSA per se does not significantly increase the cardiovascular risk, while the coexistence with a higher BMI, might be likely responsible for the worsen of the prognosis.

In two recent studies $(24,25)$, endothelial peripheral dysfunction in OSA patients has been significantly found in mild OSA and in minimally symptomatic OSA. The endothelial dysfunction decreased by 3 months of C-PAP treatment. Indeed, in our study, we demonstrated that moderate or severe OSA patients show a progressive brachial endothelial dysfunction, established by flow-mediated dilation (FMD) assay. The most evident finding was the significant reversibility of FMD in the group of patients treated with C-PAP therapy, for at least 3 months (24).

In addition, OSA-associated chronic intermittent hypoxia (CIH), in obesity, may complicate adipose tissue hypoxia with over-production of adipose tissue inflammation, by increasing macrophage infiltration and activating redox-sensitive transcription factors, as well as, ER stress signaling proteins (26). In a very recent study, it has been evaluated the effect of OSA and C-PAP on changes in adipose tissue inflammatory/hypoxia markers, there assessed by testing mRNA levels of genes related to macrophage infiltration, hypoxia, and ER stress (27). In this investigation, authors performed abdominal subcutaneous adipose tissue biopsies from OSA and non-OSA obese (BMI > 35) individuals, at baseline and after 24 weeks (T1) of weight-loss intervention plus continuous positive airway pressure (C-PAP), or weight-loss intervention alone, respectively. In obese individuals with OSA, the reduction of $\mathrm{CIH}$, by a correct C-PAP therapy, associated with a weight-loss intervention strategy, decreased mRNA expression of markers related to tissue hypoxia, reduced plasma levels of some pro-inflammatory cytokines and growth factors, ER stress, inflammation, and, finally, macrophage infiltration (27),

The endothelial, as well as, the adipose tissue dysfunction, with the consequent increased cardiovascular risk, are an early phenomenon in OSA patients, and, according to our results, show-up more severe in subjects with a BMI higher than 30, while did not appear statistically significant as compared to the degree of OSA. Thus, the current study demonstrates that newly diagnosed subjects with OSA are already at risk for cardiovascular events, and patients with higher levels of BMI, as well as with higher levels of AHI, experience also the presence of more elevated number of co-morbidities, as seen by the results of Charlson Co-morbidities Index (Table 1). In order to avoid this condition, OSA should be diagnosed as soon as possible, in patients with elevated BMI, who likely improve their endothelial dysfunction, and subsequently their cardiovascular risk with C-PAP therapy. This treatment has been shown to significantly decrease the cardiovascular risk and the development of several co-morbidities, often responsible for fatal events (24).

This study has several limitations. First, the newly diagnosed population of OSA was evaluated retrospectively. It is unknown whether these subjects had a benefit or not by using C-PAP, in terms of cardiovascular risk, as compared to them who refused or were intolerant. Second, smoking habit was highly in obese patients, which could play a significative role for increasing the cardiovascular risk. Third, the weak association between BMI and AHI and the lack of correlation between degree of OSA and increased cardiovascular risk was not well established.

In conclusion, further studies must investigate the pivotal role of systemic inflammation due to obesity, which appears to be able to provoke increased cardiovascular risk and augmented co-morbidities, in OSA patients.

Compliance with Ethical Standards: This study received no funding. 
Pierluigi Carratù declares that he has no conflict of interest

Agostino Di Ciaula declares that he has no conflict of interest

Silvano Dragonieri declares that he has no conflict of interest

Teresa Ranieri declares that she has no conflict of interest

Marco Matteo Ciccone declares that he has no conflict of interest

Piero Portincasa declares that he has no conflict of interest

Onofrio Resta declares that he has no conflict of interest

Ethical Approval: All procedures performed in the study involving human participants were in accordance with the ethical standards of the institutional and/or national research committee with the 1964 Helsinki declaration, and its later amendments or comparable ethical standards.

Informed consent was obtained from all individuals participants included in the study.

Authors' contribution

Pierluigi Carratù conceived the study, made data interpretation and wrote the manuscript.

Agostino Di Ciaula edited the manuscript, made statistical analysis, and approved the final revision of the manuscript.

Silvano Dragonieri designed the study, approved the final form of the manuscript.

Teresa Ranieri collected data, approved the final form of the manuscript.

Marco Matteo Ciccone participated to the study and approved the final revision of the manuscript.

Piero Portincasa participated to the study design, edited the manuscript, approved the final form of the manuscript.

Onofrio Resta participated to the study, approved the final revision of the manuscript.

References

1 European Guidelines on CVD Prevention in Clinical Practice 2016

Eur J Prev Cardiol. 2016 Jul;23(11):NP1-NP96. doi: 10.1177/2047487316653709

2 Dibyata Rana, Chenet Torrilus, Wiqas Ahmad, Nkechi A Okam, Tehreem Fatima, Nusrat Jahan. Obstructive Sleep Apnea and Cardiovascular Morbidities: A Review Article. Cureus 2020 Sep 13;12(9):e10424. doi: 10.7759 /cureus. 10424 .

1. Kohler M, Craig S, Pepperell JC, Nicoll D, Bratton DJ, Nunn AJ, Leeson P, Stradling JR (2013). CPAP improves endothelial function in patients with minimally symptomatic OSA: results from a subset study of the MOSAIC trial. Chest. 896-902.

2. Carratù $\mathrm{P}$, Carratù L, Resta $\mathrm{O}$. What is more affected in patients with obstructive sleep apnea: the right or the left heart? Pol Arch Med Wewn. 2016 Apr 29;126(4):217-8. doi: 10.20452/pamw.3384.

3. Kostas Archontogeorgis, Athanasios Voulgaris, Evangelia Nena, Maria Strempela, Panagiota Karailidou, Argyrios Tzouvelekis, Toulin Mouemin, Maria Xanthoudaki, Stylianos Steiropoulos, Marios E. Froudarakis, and Paschalis Steiropoulos. Cardiovascular Risk Assessment in a Cohort of Newly Diagnosed Patients with Obstructive Sleep Apnea Syndrome. Cardiology Research and Practice Volume 2018, Article ID 6572785, 8 pages

4. Carneiro G, Zanella MT. Obesity metabolic and hormonal disorders associated with obstructive sleep apnea and their impact on the risk of cardiovascular events. Metabolism. 2018 Mar 10. pii: S00260495(18)30074-X. doi: 10.1016/j.metabol.2018.03.008. 
5. Carratù P, Ventura VA, Maniscalco M, Dragonieri S, Berardi S, Ria R, Quaranta VN, Vacca A, Devito F, Ciccone MM, Phillips BA, Resta O. Echocardiographic findings and plasma endothelin-1 levels in obese patients with and without obstructive sleep apnea. Sleep Breath. 2016 May;20(2):613-9.

6. Wong CY, O'Moore-Sullivan T, Leano R, Byrne N, Beller E, Marwick TH (2004) Alterations of left ventricular myocardial characteristics associated with obesity. Circulation 110(19):3081-3087

7. Segers VF, Brutsaert DL, De Keulenaer GW (2012) Pulmonary hypertension and right heart failure in heart failure with preserved left ventricular ejection fraction: pathophysiology and natural history. Curr Opin Cardiol 27(3):273-280

8. Sharma SK, Mishra HK, Sharma H, Goel A, Sreenivas V, Gulati V, Tahir M. Obesity, and not obstructive sleep apnea, is responsible for increased serum hs-CRP levels in patients with sleep-disordered breathing in Delhi. Sleep Medicine Volume 9, Issue 2, January 2008, Pages 149-156.

9. Zamanian RT, Hansmann G, Snook S et al (2009) Insulin resistance in pulmonary arterial hypertension. Eur Respir J 33(2):318-324.

10. Reardon CA, Lingaraju A, Schoenfelt KQ, Zhou G, Cui C, Jacobs-El H, Babenko I, Hoofnagle A, Czyz D, Shuman H, Vaisar T, Becker L.

Obesity and Insulin Resistance Promote Atherosclerosis through an IFN $\gamma$-Regulated Macrophage Protein Network. Cell Rep. 2018 Jun 5;23(10):3021-3030.

Leung CC, Moondra V, Catherwood E, Andrus BW (2010) Prevalence and risk factors of pulmonary hypertension in patients with elevated pulmonary venous pressure and preserved ejection fraction. Am J Cardiol 106(2):284-286.

J-Y Xie 1, W-X Liu, L Ji, Z Chen, J-M Gao, W Chen, G-F Chen, Q Zhu; Relationship between inflammatory factors and arrhythmia and heart rate variability in OSAS patients. Eur Rev Med Pharmacol Sci 2020 Feb;24(4):2037-2053.

15 David C Goff Jr, Donald M Lloyd-Jones, Glen Bennett, Sean Coady, Ralph B D'Agostino, Raymond Gibbons, Philip Greenland, Daniel T Lackland, Daniel Levy, Christopher J O'Donnell, Jennifer G Robinson, J Sanford Schwartz, Susan T Shero, Sidney C Smith Jr, Paul Sorlie, Neil J Stone, Peter W F Wilson, Harmon S Jordan, Lev Nevo, Janusz Wnek, Jeffrey L Anderson, Jonathan L Halperin, Nancy M Albert, Biykem Bozkurt, Ralph G Brindis, Lesley H Curtis, David DeMets, Judith S Hochman, Richard J Kovacs, E Magnus Ohman, Susan J Pressler, Frank W Sellke, Win-Kuang Shen, Sidney C Smith Jr, Gordon F Tomaselli, American College of Cardiology/American Heart Association Task Force on Practice Guidelines 2013 ACC/AHA guideline on the assessment of cardiovascular risk: a report of the American College of Cardiology/American Heart Association Task Force on Practice Guidelines. Circulation 2014 Jun 24;129(25 Suppl 2):S49-73.

American Thoracic Society (1995) Standardization of spirometry,

1994 update. Am J Respir Crit Care Med 152: 1107-1136

17 Patil SP, Schneider H, Schwartz AR, et al. Adult obstructive sleep apnea: pathophysiology and diagnosis. Chest 2007; 132: 325-337.

Tetyana Kendzerska, Richard S Leung, Clare L Atzema, George Chandy, Moussa Meteb, Atul Malhotra , Gillian A Hawker, Andrea S Gershon Cardiovascular consequences of obstructive sleep apnea in women: a historical cohort study. Sleep Med 2020 Apr;68:71-79.

Papanikolaou J, Ntalapascha M, Makris D, Koukoubani T, Tsolaki V, Zakynthinos G, Gourgoulianis K, Zakynthinos E. Diastolic dysfunction in men with severe obstructive sleep apnea syndrome but without cardiovascular or oxidative stress-related comorbidities. Ther Adv Respir Dis

Jan-Dec 2019 Observational study

G Berger 1, R Berger, A Oksenberg. Progression of snoring and obstructive sleep apnoea: the role of increasing weight and time Eur Respir J. 2009 Feb;33(2):338-45 
Punjabi NM, Sorkin JD, Katzel LI, et al. Sleep-disordered breathing and insulin resistance in middle-aged and overweight men. Am J Respir Crit Care Med 2002; 165: 677-682.

22. Friedman SE, Andrus BW (2012) Obesity and pulmonary hypertension:

a review of pathophysiologic mechanisms. J Obes 50(5):

274

23 Gabriela Alarcon, Julieta Roco, Mirta Medina, Analia Medina, Maria Peral, Susana Jerez High fat dietinduced metabolically obese and normal weight rabbit model shows early vascular dysfunction: mechanisms involved. Int J Obes (Lond)

2018 Sep;42(9):1535-1543.

Bastard JP, Maachi M, Lagathu C et al (2006) Recent advances in

the relationship between obesity, inflammation, and insulin resistance.

Eur Cytokine Netw 17(1):4-12

24. Ciccone MM, Favale S, Scicchitano P, Mangini F, Mitacchione G, Gadaleta F, Longo D, Iacoviello M, Forleo C, Quistelli G, Taddei S, Resta O, Carratù P. Reversibility of the endothelial dysfunction after CPAP therapy in OSAS patients. Int J Cardiol

2012 Jul 26;158(3):383-6

25. Kohler M, Craig S, Pepperell JC, Nicoll D, Bratton DJ, Nunn AJ, Leeson P, Stradling JR (2013). CPAP improves endothelial function in patients with minimally symptomatic OSA: results from a subset study of the MOSAIC trial. Chest. 896-902

26. Hotamisligil GS. Endoplasmic reticulum stress and the inflammatory basis of metabolic disease. Cell. 2010;140(6):900-917. 27. Perrini S, Cignarelli A, Quaranta VN, Falcone VA, Kounaki S, Porro S, Ciavarella A, Ficarella R, Barbaro M, Genchi VA, Nigro P, Carratù P, Natalicchio A, Laviola L, Resta O, Giorgino F. JCI Insight. 2017 Sep 7;2(17). pii: 94379. doi: 10.1172/jci.insight.94379.

\section{Hosted file}

Figures manuscript BMI.pdf available at https://authorea.com/users/401913/articles/513864obstructive-sleep-apnea-syndrome-does-not-increase-the-cardiovascular-risk-in-ana\%C3\%AFve-population-of-southern-italy

\section{Hosted file}

Table 1 BMI manuscript.pdf available at https://authorea.com/users/401913/articles/513864obstructive-sleep-apnea-syndrome-does-not-increase-the-cardiovascular-risk-in-ana\%C3\%AFve-population-of-southern-italy 